\title{
Medical Image of the Week: Infective Endocarditis in an IV Drug User
}
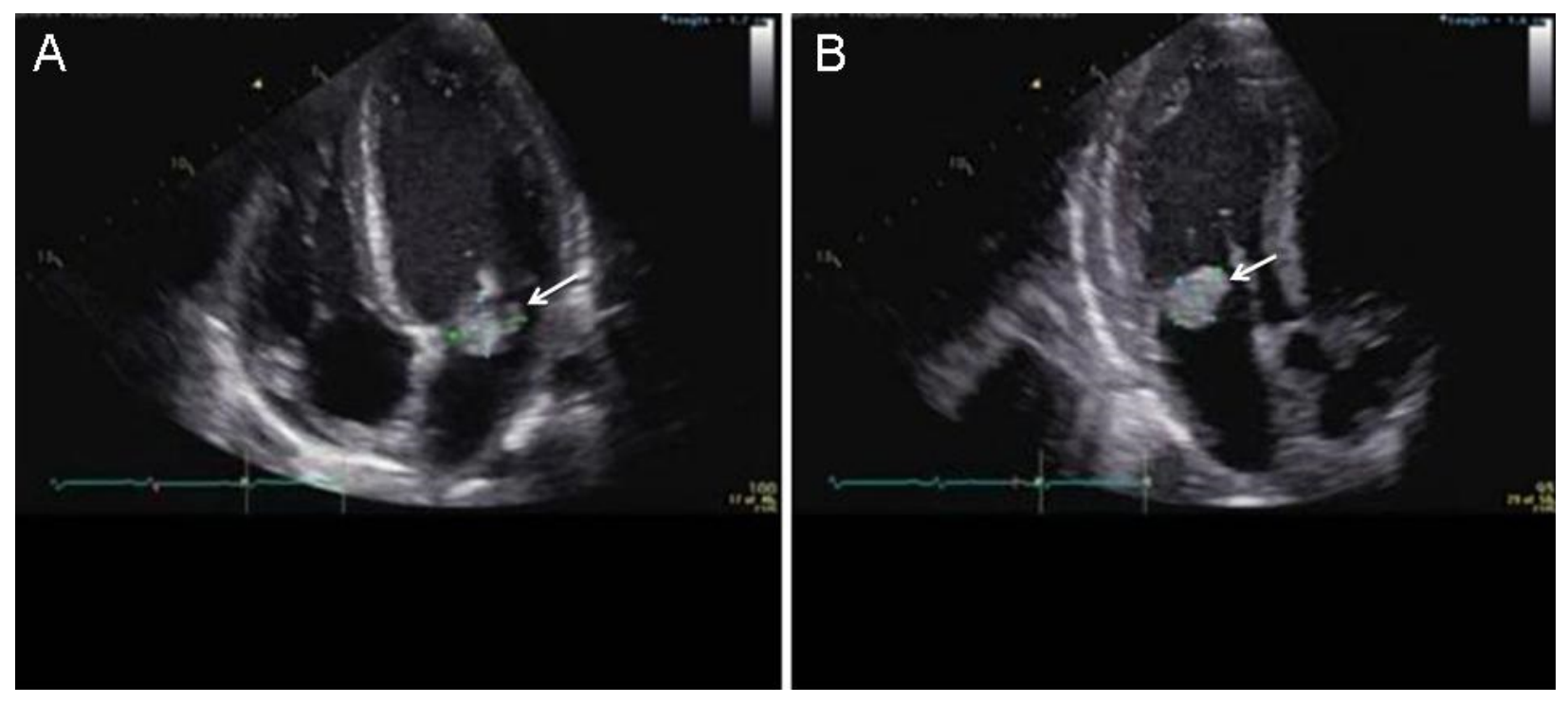

Figure 1. Transthoracic echocardiogram showing a large, irregular, mobile mass attached to the mitral valve annulus (arrows).

A 30 year old man presented with a one week history of fever, chills, body aches, and fatigue, as well as lower extremity and right wrist edema and pain. The patient also had a history of intravenous drug use. On exam, a previously undocumented $3 / 6$ blowing crescendo murmur was heard at the fifth intercostal space in the midclavicular line. Transthoracic echocardiogram demonstrated a large, irregular, mobile mass, measuring $2.0 \times 2.5 \mathrm{~cm}$, attached to the posterior mitral annulus (Figure 1). Cardiothoracic surgery performed a primary repair of the mitral valve.

Abigail S. Hawke, MD

Department of Internal Medicine

University of Arizona

Tucson, Arizona

Arthia Satyanarayan, MS III

University of Arizona College of Medicine

Tucson, Arizona 